\title{
More on Subgroup Analyses in Clinical Trials
}

TO THE EDITOR: With regard to the article by Wang et al. (Nov. 22 issue), it is increasingly recognized that subgroup analyses in clinical trials are sometimes misused and require appropriate presentation and cautious interpretation..$^{1,2}$ However, we would recommend that one type of subgroup analysis (in large "positive" trials or metaanalyses) be performed more often. That is, from a prognostic model, one categorizes patients into several ordered risk groups and estimates the absolute risk reduction in each group. For instance, the Third Randomized Intervention Treatment of Angina (RITA-3) trial, ${ }^{3}$ involving patients with acute coronary syndromes, showed an overall reduction in the risk of myocardial infarction or death associated with an invasive strategy as compared with conservative management. Such a riskstratified subgroup analysis then usefully clarified that this benefit was mainly in patients at high risk for myocardial infarction or death.

In general, such an approach can be an important aid for clinical practice, so that for any specific patient, one can judge the absolute benefit of a new treatment (set against the absolute risk of side effects, as well as costs if relevant) and decide whether the treatment is warranted for that patient. This approach is also applicable when, with regard to relative risk, there is no evidence of subgroup interactions with treatment. The question of whether prasugrel is preferable to clopidogrel in acute coronary syndromes ${ }^{4}$ is an interesting example; it may be that the absolute benefit of the reduced risk of ischemic events outweighs the increased risk of bleeding only for high-risk patients.

Similarly, if the absolute risk of myocardial infarction due to rofecoxib (Vioxx) had been properly quantified in appropriate risk-stratified subgroup analyses, the benefits of pain relief and reduced gastrointestinal bleeding might have been more clearly seen to outweigh the low absolute risk of myocardial infarction in a substantial proportion of patients, thus permitting the continued prescription of rofecoxib with appropriately restrictive labeling.

Stuart J. Pocock, Ph.D.

London School of Hygiene and Tropical Medicine

London WCIE 7HT, United Kingdom

stuart.pocock@lshtm.ac.uk

\section{Jacobus Lubsen, Ph.D. \\ SOCAR Research SA \\ $\mathrm{CH}-1260$ Nyon, Switzerland}

Dr. Pocock reports serving on paid advisory boards for Eli Lilly and Merck. No other potential conflict of interest relevant to this letter was reported.

1. Wang R, Lagakos SW, Ware JH, Hunter DJ, Drazen JM. Statistics in medicine - reporting of subgroup analyses in clinical trials. N Engl J Med 2007;357:2189-94.

2. Assmann SF, Pocock SJ, Enos LE, Kasten LE. Subgroup analyses and other (mis)uses of baseline data in clinical trials. Lancet 2000;355:1064-9.

3. Fox KAA, Poole-Wilson P, Clayton TC, et al. 5-Year outcome of an interventional strategy in non-ST-elevation acute coronary syndrome: the British Heart Foundation RITA 3 randomised trial. Lancet 2005;366:914-20.

4. Wiviott $\mathrm{SD}$, Braunwald $\mathrm{E}, \mathrm{McCabe} \mathrm{CH}$, et al. Prasugrel versus clopidogrel in patients with acute coronary syndromes. $\mathrm{N} \mathrm{Engl} \mathrm{J}$ Med 2007;357:2001-15.

THE AUthors Reply: We agree with Pocock and Lubsen about the value of subgroup analyses in examining whether the absolute risk reduction varies across a baseline risk categorization. However, obtaining and applying such information in practice pose challenges. Whenever possible, the algorithm for forming risk categories should be determined from external data. ${ }^{1}$ When a multivariate regression model is used to form risk categories that are based on the same data used to evaluate the new treatment, it is preferable to build the model only with the use of data from the subjects in the control group. Risk categories suggested by observed treatment-group differences are prone to bias and should be avoided.

It is also important to consider the robustness of the conclusions of such analyses with regard to different choices of risk categories. For example, patients in the RITA-3 trial were initially classified into four categories (1, 2, 3, and 4) and then further classified into five categories by splitting category 4 into two groups (4a and $4 b){ }^{2}$ The fivecategory classification suggests relatively small absolute treatment differences in all groups except 4b (19\%), whereas the four-level categorization suggests a large effect only in category 4 (13\%), which might lead to a different treatment strategy for patients in category 4a. Furthermore, the uncertainty in the estimated risk reduction within each risk group needs to be considered. For example, in the RITA-3 trial, the estimated risk reduction for category $4 \mathrm{a}$ is $4.1 \%$, but the corre- 
sponding $95 \%$ confidence interval ranges from a $16.7 \%$ reduction to an $8.5 \%$ increase. Finally, within-level comparisons should be preceded by evidence of the heterogeneity of treatment effect based on an interaction test oriented to differences in absolute risk.

Rui Wang, M.S.

Stephen W. Lagakos, Ph.D.

Harvard University

Boston, MA 02115
1. Rothwell PM, Mehta Z, Howard SC, Gutnikov SA, Warlow CP. Treating individuals 3: from subgroups to individuals: general principles and the example of carotid endarterectomy. Lancet 2005;365:256-65.

2. Fox KAA, Poole-Wilson P, Clayton TC, et al. 5-Year outcome of an interventional strategy in non-ST-elevation acute coronary syndrome: the British Heart Foundation RITA 3 randomised trial. Lancet 2005;366:914-20.

\section{Dual Inheritance of Sudden Death from Cardiovascular Causes}

TO THE EDITOR: The long-QT syndrome and catecholaminergic polymorphic ventricular tachycardia are the most common inherited cardiac channelopathies. ${ }^{1}$ Although the disease mechanisms for these two disorders differ, the resulting arrhythmias are similar, and both are triggered by sympathetic stimulation. ${ }^{2,3}$ We identified the coexistence of the two diseases in one family.

A family with a history of recurrent sudden death was studied. During the past 30 years, nine members have died suddenly between 7 and 40 years of age. A borderline QT interval corrected for heart rate (QTc) of $450 \mathrm{msec}$ in one asymptomatic relative (identified as V-1 in Fig. 1) was noted. Genetic testing revealed a heterozygous mutation in KCNQ1 (1343delC, p.P448fsX465), confirming the diagnosis of the long-QT syndrome type $1 .{ }^{4} \mathrm{~A}$ diagnostic molecular test for this muta- tion was therefore performed in all consenting family members.

Subsequently, one girl (identified as V-13 in Fig. 1) who did not inherit the KCNQ1 mutation died suddenly at 13 years of age. In addition, several other noncarriers had symptoms such as syncope. Resting electrocardiograms (ECGs) from these family members did not show any abnormalities. During exercise stress testing, premature ventricular beats were detected. A second familial disorder was suspected. Therefore, family members were evaluated to identify members meeting the following criteria: symptoms or sudden death during sympathetic stimulation, normal findings on a resting ECG, the absence of structural cardiac abnormalities, and premature ventricular beats during exercise.

A pedigree analysis indicated that the second

Figure 1. Key Extract of the Pedigree of Five Generations of a Family with the Long-QT Syndrome and Catecholaminergic Polymorphic
Ventricular Tachycardia.
Circles indicate female family members, squares male family members, solid symbols clinically affected family members, and open sym-
bols clinically unaffected family members. Deceased family members are indicated by slashes through the symbols. Symbols with
squares within squares and squares within circles indicate obligate carriers. Diamonds indicate persons for whom sex was unknown.
The upper symbol below a circle or square indicates the presence (+) or absence (-) of the KCNQ1 mutation, and the lower symbol indi-
cates the presence (+) or absence (-) of the RYR2 mutation in persons who underwent DNA testing. The boxed area is the part of the
family in which the KCNQ1 mutation was introduced by marriage.

